\title{
Development and deployment of KASP markers for multiple alleles of $L r 34$ in wheat
}

\author{
Tilin Fang ${ }^{1} \cdot$ Lei Lei $^{1} \cdot$ Genqiao Li $^{1}$ - Carol Powers ${ }^{1} \cdot$ Robert M. Hunger $^{2} \cdot$ Brett F. Carver $^{1} \cdot$ Liuling Yan $^{1}$ (I)
}

Received: 8 October 2019 / Accepted: 31 March 2020 / Published online: 12 April 2020

(c) The Author(s) 2020

\begin{abstract}
Key message Heterogeneous Lr34 genes for leaf rust in winter wheat cultivar 'Duster' and KASP markers for allelic variation in exon 11 and exon 22 of Lr34.

Abstract Wheat, Triticum aestivum $(2 n=6 x=42$, AABBDD), is a hexaploid species, and each of three homoeologous genomes A, B, and D should have one copy for a gene in its ancestral form if the gene has no duplication. Previously reported leaf rust resistance gene $L r 34$ has one copy on the short arm of chromosome 7D in hexaploid wheat, and allelic variation in Lr34 is in intron 4, exon 11, exon 12, or exon 22. In this study, we discovered that Oklahoma hard red winter wheat cultivar 'Duster' (PI 644,016) has two copies of the Lr34 gene, the resistance allele Lr34a and the susceptibility allele Lr34b. Both $L r 34 a$ and $L r 34 b$ were mapped in the same linkage group on chromosome 7D in a doubled-haploid population generated from a cross between Duster and a winter wheat cultivar 'Billings' which carries the susceptibility allele $\mathrm{Lr} 34 \mathrm{c}$. A chromosomal fragment including $L r 34$ and at least two neighboring genes on its proximal side but excluding genes on its distal side was duplicated in Duster. The Duster Lr34ab allele was associated with tip necrosis and increased resistance against leaf rust at adult plants in the Duster $\times$ Billings DH population tested in the field, demonstrating the function of the Duster Lr34ab allele in wheat. We have developed KASP markers for allelic variation in exon 11 and exon 22 of Lr34 in wheat. These markers can be utilized to accelerate the selection of $\operatorname{Lr} 34$ in wheat.
\end{abstract}

\section{Introduction}

Wheat, Triticum aestivum ( $2 n=6 x=42$, AABBDD), is an allopolyploid produced from two separate hybridization events. The first hybridization event occurred between the two diploid grass species T. urartu (the A genome donor, $2 n=2 x=14, \mathrm{AA}$ ) and Aegilops speltoides (the possible B genome donor, $2 n=2 x=14, \mathrm{BB})$ to form the tetraploid $T$. turgidum $(2 n=4 x=28$, AABB $)$, and the second hybridization event occurred between the tetraploid species and a diploid goatgrass species Ae. tauschii (the D genome

Communicated by Susanne Dreisigacker.

Tilin Fang and Lei Lei contributed equally to this study.

Liuling Yan

liuling.yan@okstate.edu

1 Department of Plant and Soil Sciences Department, Oklahoma State University, 368 AG Hall, Stillwater, OK 74078, USA

2 Entomology and Plant Pathology Department, Oklahoma State University, Stillwater, OK 74078, USA donor, $2 n=2 x=14$, DD) to form the hexaploid T. aestivum (McFadden and Sears 1946; Dvořák et al. 1993; Wang et al. 1997; Huang et al. 2002; Chalupska et al. 2008). Each of the three homoeologous genomes A, B, and D should have one copy for a gene in its ancestral form if the gene has no duplication or deletion. The leaf rust resistance gene $\operatorname{Lr} 34$ is a simpler name for the $L r 34 / Y r 18 / S r 57 / P m 38$ genes on the short arm of chromosome 7D in hexaploid wheat that confer non-specific resistance against fungal pathogens including leaf rust, stripe rust, stem rust, and powdery mildew, as well as barley yellow dwarf virus (Krattinger et al. 2009, 2013; Lagudah et al. 2009; Risk et al. 2013). Because of the durability and non-specificity of its resistance against multiple pathogens, Lr34 has become one of the most important disease-resistance genes in wheat worldwide. Wheat breeding programs have capitalized on naturally occurring but widespread variation for this gene, as it remains a prioritized target where fungal disease protection is critical (Spielmeyer et al. 2013; Ellis et al. 2014).

Current understanding on the Lr34 gene in hexaploid wheat is that only one copy exists on each of the three homoeologous genomes. Lr34 on the short arm of 
chromosome 7D, which hereafter is referred to as $L r 34$. Only a single copy of $L r 34$ is detected in the genome of diploid wheat species Ae. tauschii, and its homologue is found in other diploid grass species such as rice (Oryza sativa) and sorghum (Sorghum bicolor) but not in maize (Zea mays), barley (Hordeum vulgare), and Brachypodium (Krattinger et al. 2013). The Lr34gene is unique to the wheat $\mathrm{D}$ genome, and its orthologue in the A or B genome of hexaploid wheat lost function. However, the resistance of Lr34 against different diseases was detectable when it was over-expressed in cereal crops including rice (Krattinger et al. 2016), Sorghum (Schnippenkoetter et al. 2017), maize (Sucher et al. 2017), barley (Boni et al. 2018), as well as tetraploid durum wheat containing A and B genomes (Rinaldo et al. 2017).

Seven alleles, $L r 34 a$ through $L r 34 g$, have been identified for $L r 34$, and the first resistance allele in cultivars such as 'Chinese Spring' is hereafter is referred to as Lr34a (Lagudah 2011). Current understanding of allelic variation in $L r 34$ is that $L r 34 a$ gained function from natural mutations of $L r 34 b$ for the first susceptibility allele as an ancestral form in cultivars such as 'Renan' (Krattinger et al. 2009; Lagudah et al. 2009; Lagudah 2011). The Lr34 allele consists of 24 exons and 23 introns spanning 11,805-bp nucleotide sequence from the start codon to the stop codon for translation and encodes a pleiotropic drug resistance (PDR)-like adenosine triphosphate-binding cassette $(\mathrm{ABC})$ transporter (Krattinger et al. 2009). Although additional resistance or susceptibility alleles exist in genetically divergent wheat germplasm (Dakouri et al. 2010), the Lr34 gene region has been well characterized for functional differences, such that $L r 34 a$ and $L r 34 b$ can be distinguished by three polymorphisms. The first one is a single-nucleotide polymorphism (SNP) in intron 4, the second one is a codon 'TTC' encoding phenylalanine present in exon 11 in the susceptibility allele but absent in the resistance allele, and the third one is in exon 12, which is involved in encoding a tyrosine in the $\mathrm{Lr} 34 \mathrm{~b}$ protein but a histidine in the Lr34a protein. The gainof-function model of $\mathrm{Lr} 34$ from the TTC-C haplotype for the $L r 34 b$ allele to the XXX-T haplotype (X for deletion) for the $L r 34 a$ allele fits well with genotypes of several hundred wheat accessions collected worldwide (Lagudah 2011).

The $L r 34 a$ allele also lost function due to a new mutation in winter wheat cultivars such as Jagger (Cao et al. 2010; Lagudah et al. 2009; Lagudah 2011). Lr34a is believed to confer more resistance in spring wheat from which it was initially cloned (Krattinger et al. 2009). When a population of RILs from two winter bread wheat cultivars 'Jagger' and '2174' was tested in the field, a QTL was mapped in the Lr34 region that explained 18 to $35 \%$ of the total phenotypic variation in leaf rust disease severity of field-tested adult plants for three years (Cao et al. 2010). Furthermore, the reported markers for polymorphisms in exons 11 and 12 do not apply to these two winter wheat cultivars, because both
Jagger and 2174 carry the resistant haplotype at both exon 11 and exon 12 in Lr34. However, the complete sequence of the $L r 34$ gene revealed the existence of a novel mutation in exon 22 of Lr34 in Jagger (Lagudah et al. 2009; Cao et al. 2010). Due to the presence of the SNP in exon 22 causing a premature stop codon, the mutated Lr34 protein lacked 185 amino acids, including the majority of the second transmembrane domain, resulting in a non-functional form. The Jagger susceptibility allele is designated $L r 34 d$, and the 2174 resistance allele is designated $\operatorname{Lr} 34 e$ (Lagudah 2011). The point mutation resulting in the non-functional $\mathrm{Lr} 34$ in Jagger was detected in derivatives from Jagger but not in any of the spring wheat accessions (Cao et al. 2010; Lagudah et al. 2009; Lagudah 2011), suggesting that this mutation occurred during the domestication of common wheat cultivars.

A genotype carrying the susceptibility allele at any of exon 11 , exon 12 , or exon 22 , the genotype is certainly susceptible to multiple pathogens and should not be selected. However, the previously reported molecular markers were developed on the basis of restriction enzyme digestion with PCR product, which has limited high throughput screening of alleles. In the present study, we discovered that the widely adopted winter wheat cultivar 'Duster' has both $L r 34 a$ ) and $L r 34 b$. The Lr34a and Lr34bgenes in Duster were mapped to be tightly linked in a DH population developed from a cross of Duster with winter wheat cultivar 'Billings' that carries the susceptibility allele ( $L r 34 c)$ and in an F2 population developed from a cross of Duster with 2174 that carries the resistance allele ( $L r 34 e$ ). We also developed kompetitive allele-specific PCR (KASP) markers for KASP markers for allelic variation in exon 11 and exon 22 of $L r 34$ that can be extensively utilized in wheat breeding programs.

\section{Materials and methods}

\section{Parent description and doubled-haploid progeny evaluation}

Duster (PI 644,016) is a hard red winter wheat cultivar released by the Oklahoma Agricultural Experiment Station in 2006 due to its wide adaptation across the southern Great Plains of the USA (Edwards et al. 2012). Duster traces to an $\mathrm{F}_{2: 3}$ breeding line with pedigree W0405/NE78488// $\mathrm{W} 7469 \mathrm{C} / \mathrm{TX} 81 \mathrm{~V} 6187$. The original $\mathrm{F}_{2}$ population was produced in the Pioneer HRW wheat breeding program. Commercial acceptance of Duster is partly due to its consistent adult-plant resistance to leaf rust, stripe rust, and powdery mildew, and its disease-severity ratings have averaged less than $20 \%$ in breeder nurseries with natural field inoculation since 2005 in Oklahoma (Edwards et al. 2012). The resistance of Duster to multiple foliar diseases is partly due to the $L r 34$ gene that is currently in low frequency among 
HRW wheat cultivars (Kolmer 2017; Edwards et al. 2012). Duster was also reported to have $L r 3 a$ and $L r 11$ for seedling resistance (Kolmer 2017), as well as $L r 46$ and $L r 77$ for adult plant resistance (Kolmer et al. 2019). Billings (PI 656,843) is also a hard red winter wheat cultivar that was released by the Oklahoma Agricultural Experiment Station in 2009, mainly because of its excellent grain quality in combination with high yield potential (Hunger et al. 2014). Although Lr34 is absent in Billings, it is postulated to have $\mathrm{Lr} 17$ and Lr24 (Hunger et al. 2014).

A doubled-haploid (DH) population was generated from random $\mathrm{F}_{1}$ plants derived from the single cross of Duster and Billings. The DH lines were genotyped using genotyping-bysequencing (GBS) markers, and a total of 2358 GBS markers were eventually mapped in $260 \mathrm{DH}$ lines (Li et al. 2015). The sequences of GBS markers on the whole genome are archived in the NCBI SRA (accession number SRP051982). The Duster x Billings DH population was used to map the Lr34 genes in this study.

All DH lines were evaluated in the field at the Agronomy Research Station in Stillwater, OK in 2015 and 2016. The Duster $\times$ Billings DH population was arranged in the field in a replicates-in-sets design, with two replicates designed for six sets of 42 lines each but not for a seventh set of the remaining $19 \mathrm{DH}$ lines. Each set also contained the two parents, and all entries were arranged within sets as a randomized complete-block design. Under natural infection and when disease incidence appeared most pronounced, each line was evaluated for reaction to leaf rust and leaf-tip necrosis. Ratings for leaf rust and leaf-tip were independently collected on 21 May 2015, and reaction to leaf rust was recorded as two infection types, resistant for uninfected and susceptible for infected leaves of adult plants. On 6 May 2016, reaction to leaf rust was recorded based on a 1-to-4 scale, 1 for resistance showing small uredinia surrounded by necrosis, 2 for moderate resistance showing moderate size uredinia surrounded by necrosis, 3 for moderate susceptibility showing moderate size uredinia surrounded by chlorosis, and 4 for susceptibility showing large uredinia without necrosis or chlorosis.

\section{Discovery of the heterogeneous Lr34 genes in Duster}

Previously reported three SNP markers for allelic variation in exon 11 and exon 12 (Lagudah et al. 2009) and exon 22 (Cao et al. 2010) were used to genotype Duster and Billings, while Jagger and 2174 were used as controls. In this study, a new PCR marker was developed to detect a SNP in intron 4 between the susceptible $L r 34 b$ gene in Duster and $L r 34 c$ in Billings. The primers for this marker were Lr34D-In4-F1 and Lr34D-In4-R1 (Table 1). The susceptible Lr34b gene in Duster has a ' $\mathrm{T}$ ' at position $677 \mathrm{bp}$ from GT at the 5 ' end of intron 4, whereas Billings has an 'A' in the same position. The PCR products containing (TCTTC) from the susceptible $L r 34 b$ were digested with the restriction enzyme Mbo II.

In addition, a PCR marker was developed to show differences in the resistant $L r 34$ gene between Duster and 2174 . The first marker was involved a SNP at position $10,618 \mathrm{bp}$ upstream from the start codon based on the Lr34 gene sequence in Chinese Spring (FJ436983). Two primers, Lr34Prom-F2M and Lr34Prom-R2M (Table 1), were used to amplify a $111 \mathrm{bp}$ fragment followed by digestion with the restriction enzyme HpaI. After digestion, the Duster Lr34a showed $97 \mathrm{bp}$ and $14 \mathrm{bp}$, whereas the $2174 \mathrm{Lr} 34 d$ allele showed $69 \mathrm{bp}, 28 \mathrm{bp}$, and $14 \mathrm{bp}$.

All PCRs for Lr34 markers were performed using LongAmp Taq DNA polymerase (New England BioLabs) and 40 thermal cycles after denaturing at $95{ }^{\circ} \mathrm{C}$ for $5 \mathrm{~min}$, with each cycle consisting of $94{ }^{\circ} \mathrm{C}$ for $30 \mathrm{~s}, 55^{\circ} \mathrm{C}$ for $30 \mathrm{~s}$, and $72{ }^{\circ} \mathrm{C}$ for an extension time that depended on the expected sizes of the PCR products. Unless indicated otherwise in the figure legends, the resulting PCR products were run for electrophoresis on a $1 \%$ agarose gel.

\section{Isolation of Lr34-D genes by PCR}

Two primers, Cssfr6-MF2and Cssfr6-MR1 (Table 1), were designed to amplify a fragment that spanned the region from part of exon 11 to part of exon 14. The amplified PCR products were cloned into TA vectors, and four clones for each of the two alleles were sequenced.

The complete gene was isolated using two pairs of primers, ExpF1 and Cssfr6-MR1 amplifying the gene from the start codon to exon 14, and Cssfr6-MF2 and Lr34-ExpR1 amplifying the gene from exon 11 to the stop codon. The Lr34 PCR products were cloned into TA vectors (Promega), and plasmid DNAs of several individual positive colonies were sequenced. The resistant gene and the susceptibility genes were distinguishable in the overlapped region from exon 11 to exon 14, so the complete gene for each of them was assembled. The entire sequences for each gene were aligned with published Chinese Spring genome sequences of Lr34-A (TraesCS7A01G085800), Lr34-B (pseudogene), and Lr34 (TraesCS7D01G080300) to determine specific sequences for each of the homoeologous genes.

\section{Lr34 gene expression}

The total RNA was extracted from leaf samples collected from seedlings that were grown in a greenhouse with constant temperature $\left(20-25{ }^{\circ} \mathrm{C}\right)$ and long day condition $(16 \mathrm{~h} / 8 \mathrm{~h}$ for light/dark). Leaf samples were also collected from the plants that were tested in a field trial at the joining stage in February and heading stage in 2012, Stillwater Research Station, Oklahoma State University. The tip and 
Table 1 Primers and PCR markers used in the study

\begin{tabular}{|c|c|c|}
\hline Gene/locus & Primer & Primer sequence $\left(5^{\prime}-3^{\prime}\right)$ \\
\hline \multirow[t]{2}{*}{ Lr34 intron 4} & Lr34D-In4-F1 & ACGGCGCAATTGCCTTAATCCTC \\
\hline & Lr34D-In4-R1 & CACAGTGATCGCCTAGACGCC \\
\hline \multirow[t]{2}{*}{ Lr34 promoter } & Lr34Prom-F2M & AAGTTCAAGGGGTTAACTACGATGAC \\
\hline & Lr34Prom-R2M & CCGATTTTGATTAATCTCTAATCCCTAGTT \\
\hline \multirow[t]{2}{*}{ Lr34 exons 11-14 } & Cssfr6-MF2 & TCTTCAAAACAGGCCAGGTTAA \\
\hline & Cssfr6-MR1 & TACTTTCCTGAAAATAATACAAGC \\
\hline \multirow[t]{2}{*}{ Gene expression } & Lr34-E10F1 & CTCATGAATTATCAAGCATGTTCAG \\
\hline & Lr34-E14R1 & CCAATCCAAAAGCGATATAAAATAAG \\
\hline \multirow[t]{3}{*}{ Lr34-KASP-E11 } & E11r-Forward & FAM-GGGAGCATTATTTTTTTCCATCA \\
\hline & E11s-Forward & HEX-GGGAGCATTATTTTTTTCCATCT \\
\hline & Reverse & AGCGAATCCAGTATGGAAAT \\
\hline \multirow[t]{3}{*}{ Lr34-KASP-E22 } & E11r-Forward & FAM-AATGTATCGTGAGAGATTTGCA $\boldsymbol{G}$ \\
\hline & E11r-Forward & HEX-AATGTATCGTGAGAGATTTGCAT \\
\hline & Reverse & AGGTGAATAAATATGAGCATCAGT \\
\hline \multirow[t]{2}{*}{ TraesCS7D01G080100 } & Forward & GATCTGCTGTAGAGATAGCTA \\
\hline & Reverse & CACTGTCAATGAATAGACAGAAA \\
\hline \multirow[t]{2}{*}{ TraesCS7D01G080200 } & Forward & AGCGTCGTCGGCGTCTC \\
\hline & Reverse & GCAGGAAAGGCTCCATGGACA \\
\hline \multirow[t]{2}{*}{ TraesCS7D01G080300 } & Forward & AAGCATCTAACATCATTGCTG \\
\hline & Reverse & CCAATCCAAAAGCGATATAAAATAAG \\
\hline \multirow[t]{2}{*}{ TraesCS7D01G080400 } & Forward & CGGATCTTCCTGCAGCTA \\
\hline & Reverse & GTCCTTGGTGAAGATGAATGCA \\
\hline \multirow[t]{2}{*}{ TraesCS7D01G080500 } & Forward & GATCGAGGAAGACGGC \\
\hline & Reverse & AGCACTAGAGAGACCTCC \\
\hline
\end{tabular}

SNPs between alleles are highlighted in bold italics base tissues of the flag leaves from adult plants were separated to extract RNAs.

In order to test if two Lr34 genes in Duster were expressed, two specific primers Lr34-E10F1 and Lr34E14R1 (Table 1) were simultaneously used to amplify Lr34 transcripts containing exon 11 and exon 12, where the resistance and susceptibility alleles can be distinguished by using the restriction enzyme Fnu4HI. The PCR was performed by using LongAmp Taq DNA polymerase (New England BioLabs) and 35 thermal cycles after denaturing at $95^{\circ} \mathrm{C}$ for $5 \mathrm{~min}$, with each cycle consisting of $94^{\circ} \mathrm{C}$ for $30 \mathrm{~s}, 55^{\circ} \mathrm{C}$ for $30 \mathrm{~s}$, and $72{ }^{\circ} \mathrm{C}$ for $90 \mathrm{~s}$. Lr34ExpF1 and Lr34ExpR1 were paired to amplify $\mathrm{Lr} 34$ transcripts.

\section{Chromosomal fragment duplication by copy number of genes.}

Lr34 was cloned by using a physical contig of BAC clones (GenBank accession number: FJ436983) (Krattinger et al. 2009). The corresponding genes in the physical contig have been annotated in the wheat genome sequence at IWGSC RefSeq v1.0 (https://urgi.versailles.inra.fr/blast/?dbgro up=wheat_iwgsc_refseq_v1_chromosomes), where Lr34 is annotated as TraesCS7D01G080300. Specific primers
(Table 1) were designed for five genes, including $L r 34$, two neighboring genes on its distal side (TraesCS7D01G080100 and TraesCS7D01G080200) and two neighboring genes on its proximal side(TraesCS7D01G080400 and TraesCS7D01G080500). Quantitative RT-PCR (qRT-PCR) was used to determine copy number of each gene by the SYBR Green PCR Master Mix, and TaCO2 was used as an endogenous control. Sequence information for these primers is provided in Table 1, and primers for $\mathrm{TaCO} 2$ control were cited from previous studies (Díaz et al. 2012; Li et al. 2015). Genome DNAs of Duster and Billings were used as PCR template. The qRT-PCRs were carried out on a CFX96 ${ }^{\mathrm{TM}}$ Real-Time system (Bio-Rad laboratories, Hercules, CA) using SsoAdvanced Universal SYBR Green Supermix (Bio-Rad laboratories, Hercules, CA). Assays were tested in 96-well formats and set up as $10 \mu \mathrm{l}$ reactions ( $3 \mu \mathrm{l}$ gDNA at $35 \mathrm{ng} / \mu \mathrm{l}, 5 \mu \mathrm{l}$ of SsoAdvanced Universal SYBR Green Supermix, $0.3 \mu \mathrm{l}$ of $10 \mathrm{mM}$ forward primer, $0.3 \mu \mathrm{l}$ of $10 \mathrm{mM}$ reverse primer, and $\left.1.4 \mu \mathrm{l} \mathrm{H}_{2} \mathrm{O}\right)$. PCR program was: denatured at $95{ }^{\circ} \mathrm{C}$ for $3 \mathrm{~min}$, with 39 cycles $\left(95^{\circ} \mathrm{C}\right.$ for $30 \mathrm{~s}$; $57{ }^{\circ} \mathrm{C}$ for $30 \mathrm{~s} ; 72{ }^{\circ} \mathrm{C}$ for $30 \mathrm{~s}$ ) for melt curve analysis from $65{ }^{\circ} \mathrm{C}$ to $95{ }^{\circ} \mathrm{C}$ with $0.5^{\circ} \mathrm{C}$ increments for $5 \mathrm{~s}$ per step. Six technical repeats were performed for each sample. 


\section{Development of KASP-based assays for multiple alleles of $\operatorname{Lr} 34$}

Four variation sites in intron 4, exon 11, exon 12, and exon 22 have been reported (Krattinger et al. 2009; Lagudah et al. 2009; Cao et al. 2010). KASP markers were tested for each of these variation sites, but only two KASP markers were finally developed for $\operatorname{Lr} 34$ : one for allelic variation in exon 11 and the other for allelic variation in exon 22.

The Duster $\times$ Billings and Duster $\times 2174 \mathrm{~F}_{2}$ lines were developed for mapping of the heterogeneous $L r 34$ in Duster. DNAs of heterozygous alleles for $L r 34$ in the $\mathrm{F}_{2}$ lines of Duster $\times$ Billings and Duster $\times 2174$ developed in this study and Jagger $\times 2174$ used in the previous study (Cao et al. 2010) were also used to validate the KASP markers.

KASP primers were designed following standard KASP guidelines (LGC Genomics, Hoddesdon, UK), and the primer sequences are provided in Table 1. The allele-specific primers were designed carrying the standard FAM (5'-GAA GGTGACCAAGTTCATGCT-3') and HEX (5'-GAAGGT CGGAGTCAACGGATT-3') tails and with the targeted SNP at the 3' end. A common genome specific primer was designed, and the total amplicon length was $174 \mathrm{bp}$ for Lr34-E11-KASP and 130 bp for Lr34-E22-KASP excluding FAM and HEX. The primer mixture comprised $46 \mu 1$ ddH2O, $30 \mu \mathrm{l}$ common primer $(100 \mu \mathrm{M})$, and $12 \mu \mathrm{l}$ of each tailed primer $(100 \mu \mathrm{M})$. Assays were tested in 96-well formats and set up as around $10 \mu \mathrm{l}$ reactions (4.83 ul DNA at $40 \mathrm{ng} / \mu \mathrm{l}, 5 \mu \mathrm{l}$ of $1 \times \mathrm{KASP}$ master mixture, and $0.14 \mu \mathrm{l}$ of primer mixture).

For Lr34-E11-KASP, PCR cycling was performed using the following protocol: hot start at $94{ }^{\circ} \mathrm{C}$ for $15 \mathrm{~min}$, followed by ten touchdown cycles $\left(94^{\circ} \mathrm{C}\right.$ for $20 \mathrm{~s}$; touchdown at $61{ }^{\circ} \mathrm{C}$ initially and decreasing by $-0.6{ }^{\circ} \mathrm{C}$ per cycle for $60 \mathrm{~s})$, followed by 45 additional cycles $\left(94{ }^{\circ} \mathrm{C}\right.$ for $20 \mathrm{~s} ; 55^{\circ} \mathrm{C}$ for $60 \mathrm{~s}$ ). For Lr34-E22-KASP, PCR was performed in the same program as did for the InDel in exon 11 of $L r 34$, except 30 additional cycles at the last step. Three replicates for each genotype were performed.

\section{Results}

\section{Discovery of two Lr34 genes in Duster}

Three published markers for variation in exons 11,12 , and 22 of $\operatorname{Lr} 34$ are routinely used to genotype germplasm in the Oklahoma State University wheat breeding program. Surprisingly but consistently, when the marker for allelic variation in exon 11 was used to genotype, Duster showed both the larger DNA fragment representing the Lr34aallele for resistance and the smaller DNA fragment representing the $L r 34 b$ allele for susceptibility (Fig. 1A). Similarly,

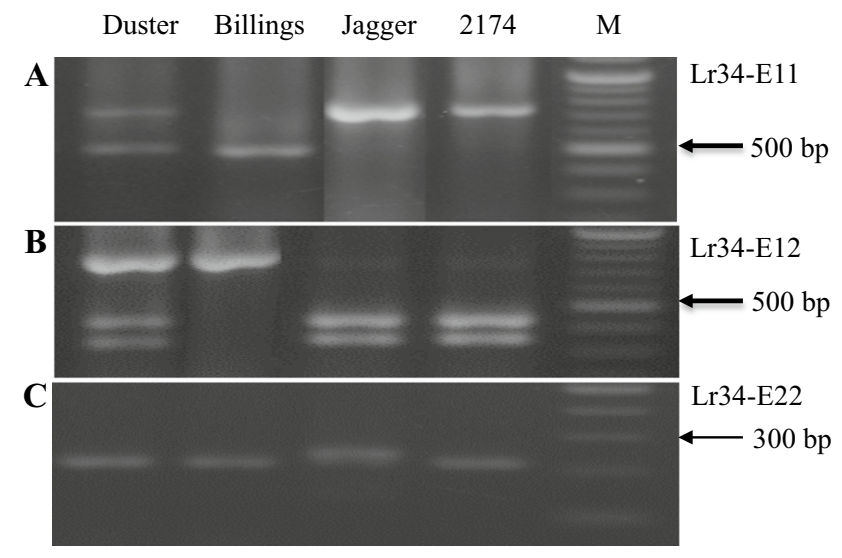

Fig. 1 Heterogeneous Lr34-D genes in Duster. (A) PCR marker for exon 11. (B) PCR marker for exon 12. (C) PCR marker for exon 22. $\mathrm{M}$ : indicates a DNA marker. Duster in the first lane shows the presence of heterogeneous $\mathrm{Lr} 34 \mathrm{genes}$ for all of the three markers. Billings shows a susceptibility allele at exon 11 and exon 12, Jagger shows a susceptibility allele at exon 22, and 2174 shows a resistant allele at each of the three markers. $\mathrm{M}$ indicates marker for $100 \mathrm{bp}$ ladder

when the marker for allelic variation in exon 12 was tested, Duster showed both the double smaller DNA fragments representing the $L r 34 a$ allele for resistance and the larger DNA fragment representing the $L r 34 b$ allele for susceptibility (Fig. 1B). When the PCR marker for the polymorphism in exon 22 was tested, Duster showed the Lr34 allele for resistance only (Fig. 1C). All other cultivars, including Jagger, 2174, and Billings, tested by the three Lr34 markers showed the same homozygosity and homogeneity as expected. As shown in Fig. 1A and B), the DNA fragments for the resistance allele and the susceptibility allele of $\operatorname{Lr} 34$ had similar intensity, suggesting the heterozygous pattern of the $\operatorname{Lr} 34$ gene in Duster. Genotyping of DNAs from individual plants of Duster, even from different seed sources, produced consistent results for the Lr34 markers, supporting the notion that the observed heterozygosity in Duster was not caused by genetic background impurity.

In order to exclude any possibility that the heterogeneous PCR products were not from $L r 34$ but from homoeologous Lr34-A or Lr34-B gene in Duster, primers Cssfr6-MF2 and Cssfr6-MR1 were used to amplify a Lr34 fragment that covered both exon 11 and exon 12 from Duster, and four clones were sequenced. Two clones representing $L r 34-D a$ showed the same sequence as the XXX-T haplotype (X for deletion) for the Lr34aallele, whereas two clones representing $L r 34 b$ showed the same sequence as the TTC-C haplotype for the $L r 34 b$ allele. These results supported that Duster had two copies of Lr34. The two Lr34 genes in Duster were temporarily designated $L r 34 a$ for the resistance gene and $L r 34 b$ for the susceptibility gene. It was intriguing to know where $L r 34 a$ and $L r 34 b$ are located in the Duster genome and why Duster had heterogeneous Lr34 genes. 


\section{Genetic mapping of two Lr34 genes}

The most convincing evidence for the existence of the $L r 34 a$ and $L r 34 b 1$ genes in Duster was that the presence or absence of each of the $L r 34 a$ and $L r 34 b$ genes was mapped in the Duster $\times$ Billings population consisting of $260 \mathrm{DH}$ lines. The DH population was generated using Duster to cross with Billings ( $\mathrm{Li}$ et al. 2015). Duster carries a resistance gene $L r 34 a$, which was confirmed in this study (Fig. 1A-C), whereas Billings carries a $\mathrm{Lr} 34$ gene for susceptibility (Hunger et al. 2014). Two PCR markers were used to map Lr34 in the DH population.

The first PCR marker was used to map the $L r 34 a$ gene. As shown in Fig. 1A, Lr34-E11 is a dominant marker (the upper band on Duster, Jagger and 2174) that was present in Duster but absent in Billings. This marker was used to genotype the $260 \mathrm{DH}$ lines, 129 lines were found to have the Lr34a gene, and the remaining 131 lines did not have this gene. The second marker is a PCR marker that was developed based on a SNP in intron 4 to map the Lr34bgene. This SNP was mapped using primers Lr34-D-In4-F1 and Lr34-D-In4-R1 to amplify a fragment, which was digested with the restriction enzyme $M b o$ II, resulting in polymorphic DNA fragments (Fig. 2A). Duster has both resistance allele (the undigested upper band) and susceptibility allele (the two digested lower bands), whereas Billings has the resistance allele (Fig. 2A). Overall, the susceptible intron 4 in Duster was tightly linked with the $\mathrm{Lr} 34 \mathrm{a}$ gene that was mapped in the 129 lines using the first marker Lr34-E11. These results indicated that Duster has both the resistance and susceptibility alleles at both intron 4 and exon 11, while Billings has the resistance allele in intron 4 but the susceptibility allele in exon 11. The two genes in Duster are tightly linked in the $260 \mathrm{DH}$ lines of Duster $\times$ Billings.

The sequence of the Lr34 gene was used to search in the recently released IWGS databases of Chinese Spring genomic sequences, IWGSC RefSeq v1.0 (IWGSC 2018), allowing the determination of this gene position from $48,964,777$ bp to $48,952,973$ chromosome 7DS. A total of 2358 GBS markers were mapped in the Duster $\times$ Billings population of $260 \mathrm{DH}$ lines, including two unlinked clusters of GBS markers, cluster 18 (28 markers) and cluster 25 (16 markers) that were assembled and mapped on chromosome 7D (Fig. 3A). Sequences of the GBS markers were used to search in the IWGS databases, allowing the determination of cluster 18 on chromosome 7DS and cluster 25 on chromosome 7DL. For example, GBS08487 included in cluster 18 was found to have $100 \%$ identity to CS sequence, and it is a single copy located from $6,026,380 \mathrm{bp}$ to $6,026,442 \mathrm{bp}$ on chromosome 7D. GBS05254 included in cluster 25 was found to have $100 \%$ identity to CS sequence, and it is a single copy located from $91,742,616$ bp to $91,742,679$ bp on chromosome 7D (Fig. 3A). However, Lr34 was mapped

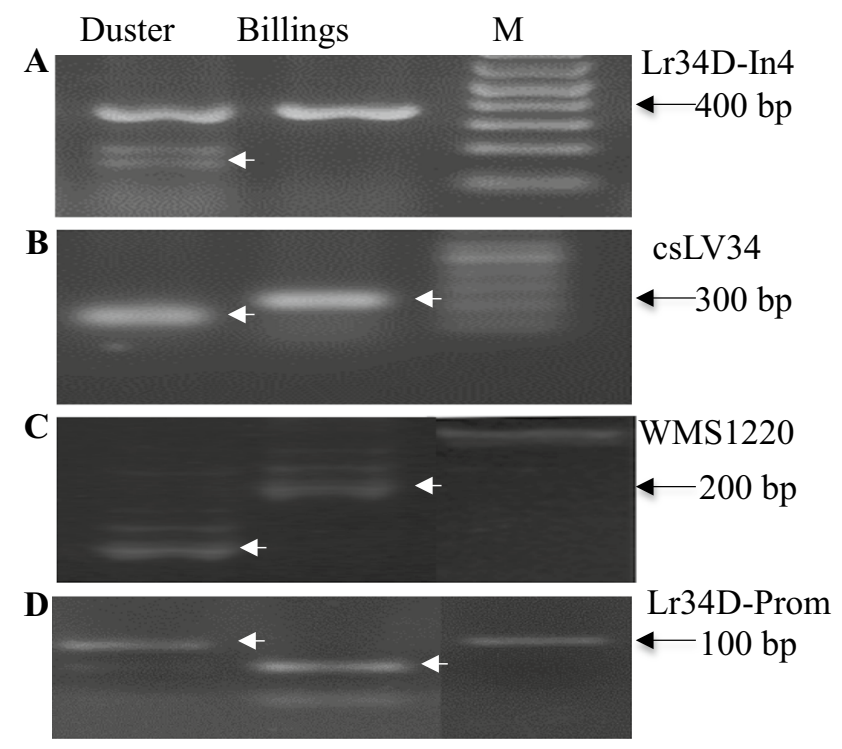

Fig. 2 PCR markers for Lr34a and Lr34b in Duster. (A) PCR marker for a SNP in intron 4. The Mbo II-digested PCR products showed $360 \mathrm{bp}$ and 157 bp-203 bp for the Duster Lr34b but only $360 \mathrm{bp}$ for the BillingsLr34a. (B) csLV34 marker. (C)WMS1220 marker. (a-c) were for allelic variation between Duster and Billings. (D) PCR marker for a SNP in the promoter region of Lr34 between Duster $L r 34 a$ and $2174 L r 34 c$. The HpaI-digested PCR products showed $97 \mathrm{bp}$ and $69 \mathrm{bp}$ for the Duster allele but $69 \mathrm{bp}$ for the 2174 allele. In addition, $14 \mathrm{bp}$ and $28 \mathrm{bp}$ are in both of the alleles. M indicates marker for $100 \mathrm{bp}$ ladder. Arrows point to DNA fragments that are mapped

$25 \mathrm{cM}$ to cluster 18 and $35 \mathrm{cM}$ to cluster 25 (Fig. 3A). These results suggested that the linked $L r 34$ genes in an approximate $85 \mathrm{Mb}$ region where no GBS markers were observed.

In order to ensure that the $\mathrm{Lr} 34$ genes are indeed located on chromosome 7DS, several markers that are reported to reside at the $L r 34$ locus were screened for polymorphisms between Duster and Billings. Two markers were polymorphic, one for $\operatorname{csLV} 34$ (Fig. 2B), which is a STS marker linked with $\operatorname{Lr} 34$ (Lagudah et al. 2006), and the other for Xgwm1220 (Fig. 2C), which is a SSR marker linked with Lr34 (Lillemo et al. 2013). Among the first 96 DH lines, crossovers were found between csLV34 and Lr34ain two DH lines (DH15 and DH39) and no crossover was found between Xgwm1220 and Lr34a, further confirming that the duplicated $L r 34 a$ and $L r 34 b$ genes were located on chromosome 7DS in Duster.

The genomic sequences for each of $L r 34 a$ and $L r 34 b$ were determined by using primers to amplify two overlapping fragments from each gene. The first fragment extending from the start codon to exon 16 was amplified using primers Lr34D-ExpF1 and Cssfr6-MR1. The second fragment extending from exon 11 to the stop codon was amplified using primers Cssfr6-MF2 and Lr34D-ExpR1. As shown in Fig. 3B, sequencing of the complete genes from start codon 


A GBS08487
GBS cluster 18
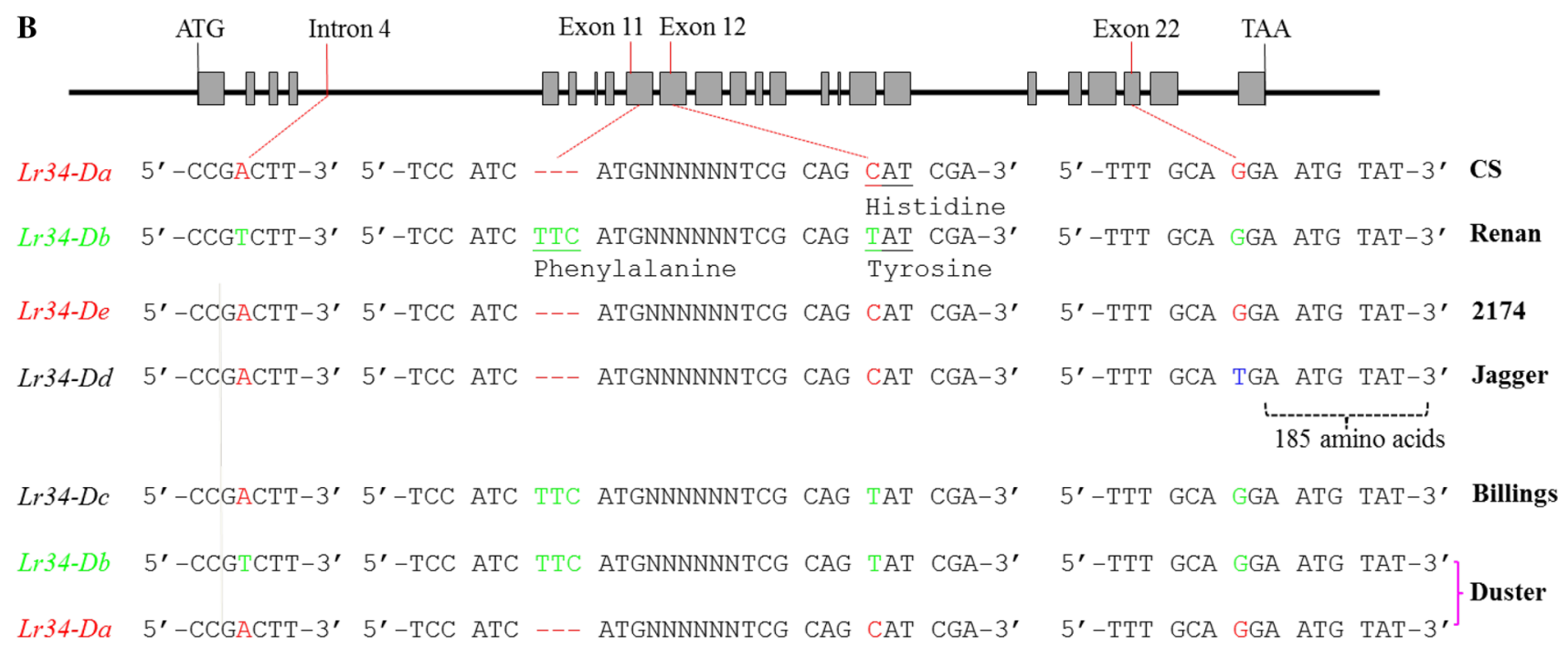

Fig. 3 Diagram of haplotypes at Lr34. (A) Location of the Duster $L r 34 a$ and $L r 34 b$ genes on chromosome 7D. The two genes are linked with GWM1220 and csLV34 but separated from the two GBS markers clusters (18 and 25). The physical location of two GBS markers, GBS08487 and GBS05254, is indicated. (B) Comparison between

to stop codon for translation showed that Duster had both $L r 34 a$ of In $4 r-E 11 r-E 12 r-E 22 r$ and Lr34b of In $4 s-E 11 s-$ $E 12 s-E 22 s$ in the same sequences as previously reported (Krattinger et al. 2009; Lagudah et al. 2009). Lr34a was indicative of the $I n 4 r$-E11r-E12r-E22r allele for resistance, and $L r 34 b$ was indicative of theIn $4 s-E 11 s-E 12 s-E 22 r$ allele for susceptibility. Billings had the same allele as $L r 34 c$, which is the structure of In $4 r-E 11 s-E 12 s-E 22 r$ (Lagudah et al. 2009). The haplotype of Lr34in Duster was hereafter referred to as the $L r 34 a b$ allele for its linked $L r 34 a / L r 34 b$ genes.

\section{Allelic variation between Lr34a and Lr34C}

Since the Lr34a in Duster and Lr34c in 2174 have identical sequences from the start codon to the stop codon for translation (Fig. 3B), all of the Lr34 markers produced the same pattern for Lr34a in Duster and $L r 34 c$ in 2174. More regions at the promoter and the $3^{\prime}$ end were sequenced, and PCR markers were developed to distinguish $L r 34 a$ in Duster and $L r 34 c$ in 2174 . Approximately $10 \mathrm{~kb}$ for the upstream of the $5^{\prime}$ end and downstream of the $3^{\prime}$ end of $L r 34$ was cloned and sequenced for each of Duster and 2174. A SNP in the upstream at position $10,540 \mathrm{bp}$ from the start codon different Lr34alleles. The sequences around four reported polymorphic sites in intron 4, exon 11, exon 12, and exon 22 are provided. The names of the alleles are cited (Lagudah 2011). The position of the premature stop codon TGA resulting in a lack of 185 amino acids in Jagger is indicated

was found between Lr34a in Duster and Lr34ein 2174, and a PCR marker for this SNP was developed using primers Lr34Prom-F2M and Lr34Prom-R2M (Fig. 2D). Among 196 $\mathrm{F}_{2}$ lines of Duster $\times 2174$, both $L r 34 a$ and $L r 34 b$ in Duster were mapped to be allelic to $L r 34 e$ in 2174 .

\section{Functional characterization of Lr34ab in Duster}

In order to determine if either or both of the $\operatorname{Lr} 34 a$ and $L r 34 b$ genes in Duster were expressed, the $L r 34$ genes were tested at the transcriptional level in leaf samples collected at different stages. As shown in Fig. 4A, Lr34c in Billings and $L r 34 b$ in Duster (the larger DNA fragment) showed similar expression patterns at different stages. Duster showed expression of additional Lr34agene (the middle and smaller DNA fragments) that was distinguished by digestion of the same cDNA samples containing $L r 34 b$ with a restriction enzyme Fnu4HI (Fig. 4A). Sequencing cDNA clones from Duster also confirmed the presence of both $L r 34 a$ and $L r 34 b$ in the Duster RNA samples.

Compared with the Billings $2 r 34 c$ allele, the Duster $L r 34 a b$ allele showed increased protection against leaf rust, when the population of Duster $\times$ Billings DH lines 


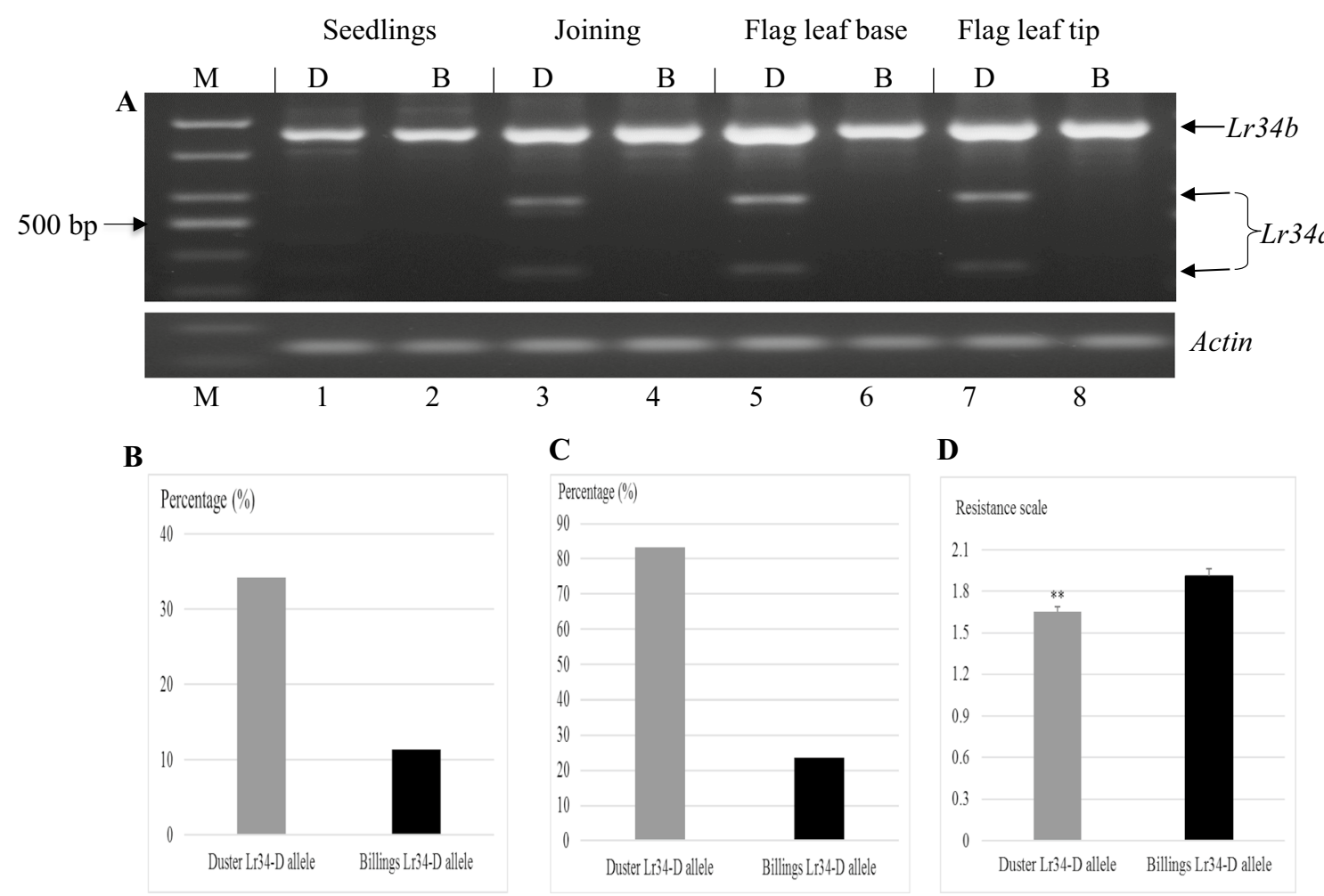

Fig. 4 Comparison of Lr34 function between Duster and Billings. (A) Expression of Lr34 at different stages. Duster (D) and Billings (B) at seedlings, leaves at joining stage, flag leaf base, flag leaf tip. Billings has Lr34c only, whereas Duster has Lr34a and Lr34d that was digested with Fnu4HI. Actin was used an endogenous gene for control. (B) Duster allele versus the Billings allele in reaction to leaf rust. Values for $Y$ axis are percentage of the resistance lines to the total DH lines carrying the Duster Lr34 allele or the Billings Lr34 allele. (C) Duster allele versus to Billings allele in tip necrosis. Values for $Y$ axis

was tested in the field in 2015. In 114 DH lines carrying the Duster Lr34ab allele, $34.2 \%$ of them had no leaf rust pustules and $65.8 \%$ of them were infected. Among $114 \mathrm{DH}$ lines carrying the Billings $\mathrm{Lr} 34 \mathrm{c}$ allele, only $11.4 \%$ of them had no leaf rust pustules and $88.6 \%$ of them were infected (Fig. 4B). The appearance of leaf-tip necrosis, indicative of $\mathrm{Lr} 34$, also showed significant difference between the Duster allele and the Billings allele; and up to $83.4 \%$ of the $114 \mathrm{DH}$ lines carrying the Duster $L r 34 a b$ allele showed the appearance of leaf-tip necrosis (Fig. 4C). When the population of Duster $\times$ Billings DH lines was tested in the field in 2016, the DH lines carrying the Duster $L r 34 a b$ allele and the DH lines carrying the Billings $L r 34 c$ gene also showed significant difference in response to leaf rust at the adult plants. Respective mean ratings for the two RILs groups were 1.65 for the Duster allele and 1.9 for the Billings allele $(p<0.0001)$, indicating that the Duster allele had increased protection against leaf rust (Fig. 4D). are numbers of plants; $Y$ is percentage of the lines with tip necrosis to the total DH lines carrying the Duster Lr34 allele or the Billings Lr34 allele. (D) Comparison of the Duster allele versus the Billings allele in reaction to leaf rust, rated on, 6 May 2016, using a 1-to-4 scale. Values for $Y$ axis are the average ratings of DH lines that have the same $\operatorname{Lr} 34$ allele to leaf rust, $n=138$ for the Duster allele and $n=124$ for the Billings allele. The bar indicates standard error; asterisk indicates that the difference is highly significant $(p<0.0001)$

\section{Duplication of a chromosomal fragment covering Lr34}

In order to determine if a single gene $\mathrm{Lr} 34$ or a chromosomal region containing in $L r 34$ Duster was duplicated, a qRT-PCR method was used to successfully determine copy number of genes in the chromosomal region containing $\operatorname{Lr} 34$. As shown in Fig. 5, Lr34 and all four neighboring genes in Billings showed one copy. In Duster, Lr34 and the two genes on its proximal side to the centromere showed two copies, but the two genes on the distal side of Lr34 showed only one copy. This result indicated that the chromosomal fragment duplication including Lr34 and at least the two neighboring genes on its proximal side occurred in Duster but not in Billings.

\section{Development of KASP-based assays for multiple alleles/haplotypes of $L r 34$}

Two KASP markers, Lr34E11-KASP and Lr34-E22-KASP, were developed to distinguish multiple alleles of $L r 34$ in 


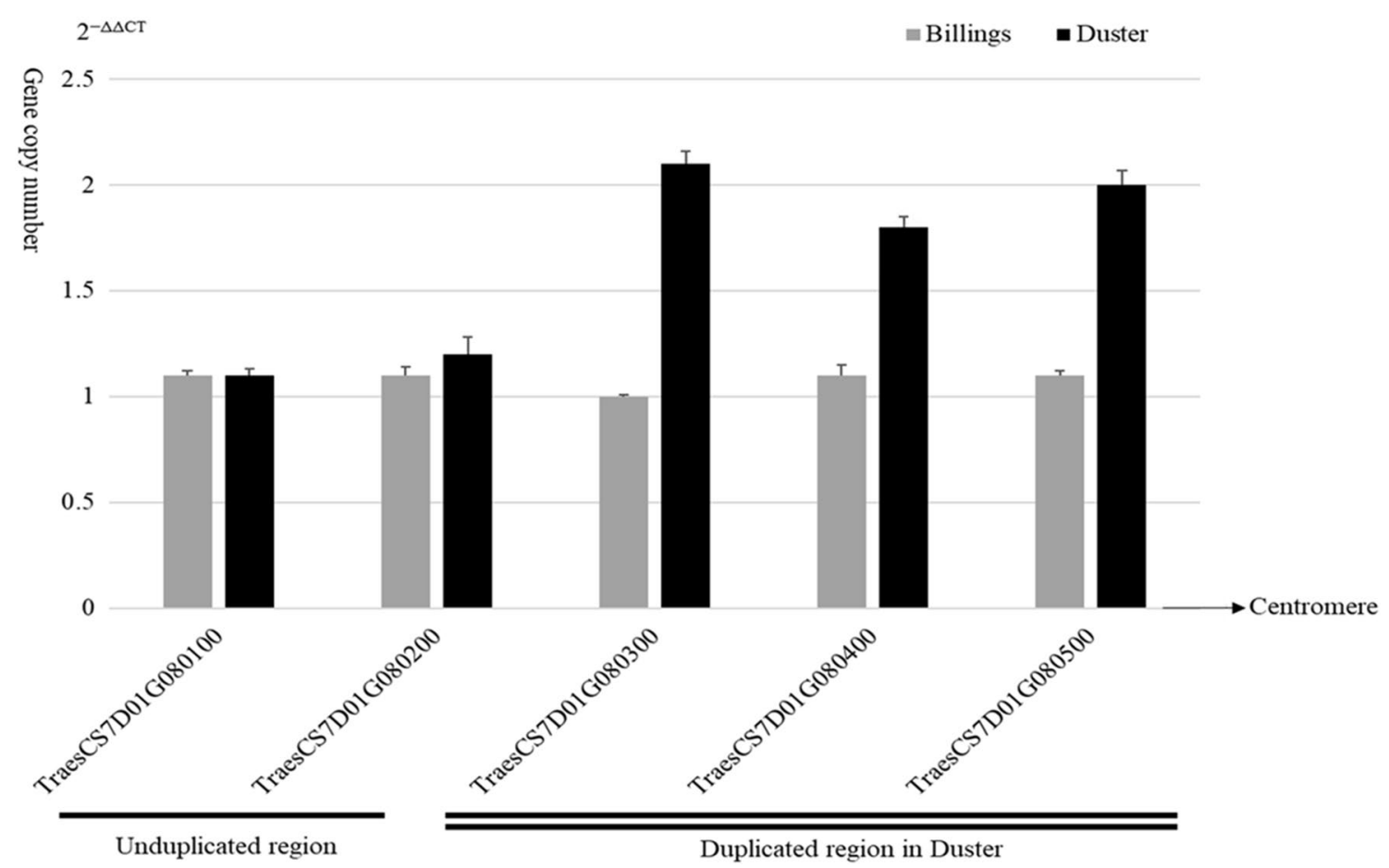

Fig. 5 A chromosomal fragment duplication event in Duster but not in Billings. qRT-PCRs were used to determine copy number of five genes including $\mathrm{Lr} 34$, two neighboring genes on its distal side (TraesCS7D01G080100 and TraesCS7D01G080200) and two neighboring genes on its proximal side (TraesCS7D01G080400 and TraesCS7D01G080500). The centromere is pointed out with an arrow. Genome DNAs of Duster and Billings were used as qRT-PCR template. $\mathrm{TaCO} 2$ was used as an endogenous control. Copy number

wheat. The forward primer for Lr34-E11-KASP has specific nucleotide at its 3 ' end, ' $T$ ' that is the first nucleotide of 'TTC' encoding phenylalanine present in exon 11 in the susceptibility allele (E11s) but 'A' that is located after the deleted 'TTC' in the resistance allele (E11r). As shown in Fig. 6A, Lr34-E11-KASP was used to effectively distinguish the susceptibility allele being in Billings and the resistance allele being in Jagger and 2174. Duster, carrying heterogeneous E11r and E11s, showed the same pattern as the heterozygous alleles of Duster (E11r and E11s) $\times$ Billings (E11s only), indicating that one copy Lr34-E11s in Duster was not distinguishable from two Lr34-E11s copies in the heterozygous Duster $\times$ Billings allele at $L r 34$. Duster also showed the same pattern as the heterozygous alleles of Duster (E11r and E11s) $\times 2174$ (E11r only), indicating that one copy Lr34E11r in Duster was not distinguishable from two Lr34-E11r copies in the heterozygous Duster $\times 2174$ allele at $L r 34$.

The forward primer for Lr34-E22-KASP has a specific nucleotide at its 3 ' end, ' $T$ ' that is the first nucleotide of 'TGA' encoding a stop codon present in exon 22 in the susceptibility allele (E22s) but ' $G$ ' in the same position for the resistance allele (E22r). As shown in Fig. 6B, is shown using the values calculated by the $2^{(-\Delta \Delta C T)}$ method, where $\mathrm{CT}$ is the threshold cycle. Bar indicates standard error. The single line under TraesCS7D01G080100 and TraesCS7D01G080200 indicates that the two genes have one copy in both Billings and Duster. The double lines under TraesCS7D01G080300, TraesCS7D01G080400, and TraesCS7D01G080200 indicate that each of these three genes has two copies in Duster (black graph) but one copy in Billings (gray graph)

Lr34-E22-KASP was used to effectively distinguish the resistance allele present in 2174, Duster and Billings and the susceptibility allele present in Jagger. Lr34-E22-KASP showed a single allele in exon 22 in Duster, compared with the heterozygous allele in Jagger $\times 2174$.

The Duster $L r 34 a b$ allele was surveyed for its frequency among parental lines used for the US T-CAP populations, recently released wheat cultivars in the southern Great Plains and relevant germplasm, and current OSU breeding lines (Fang et al. 2011). Except Duster, no other cultivar tested was found to have $L r 34 a b$.

\section{Discussion}

Lr34 has been extensively studied as a single gene since it was reported. In this study, we discovered that Duster had the new resistance allele $L r 34 a b$ allele containing both $L r 34 a$ and $L r 34 b$ genes, which are duplicated or heterogeneous. In addition to genetic confirmation of seed purity, a DH population and an $\mathrm{F}_{2}$ population were used to map the two Lr34 genes in Duster. PCR markers for presence or absence 
Fig. 6 KASP markers for Lr34. (A) Lr34-E11-KASP for allelic variation in exon 11 . (B) Lr34-E22-KASP for allelic variation in exon 22. DxB is DNA from the heterozygous allele of Duster and Billings that was confirmed using the PCR marker for allelic variation in exon 11 of Lr34. Dx2 is DNA from the heterozygous allele of Duster and 2174 that was confirmed using the PCR marker for allelic variation in exon 11 of $L r 34$. Jx2 is DNA from the heterozygous allele of Jagger and 2174 that was confirmed using the PCR marker for allelic variation in exon 22 of $\operatorname{Lr} 34$. Scatter dots with different colors show clustering of genotypes on the $X$ - (FAM) and $Y$ - (HEX) axes. Green dots represent the NTC (non-template control)
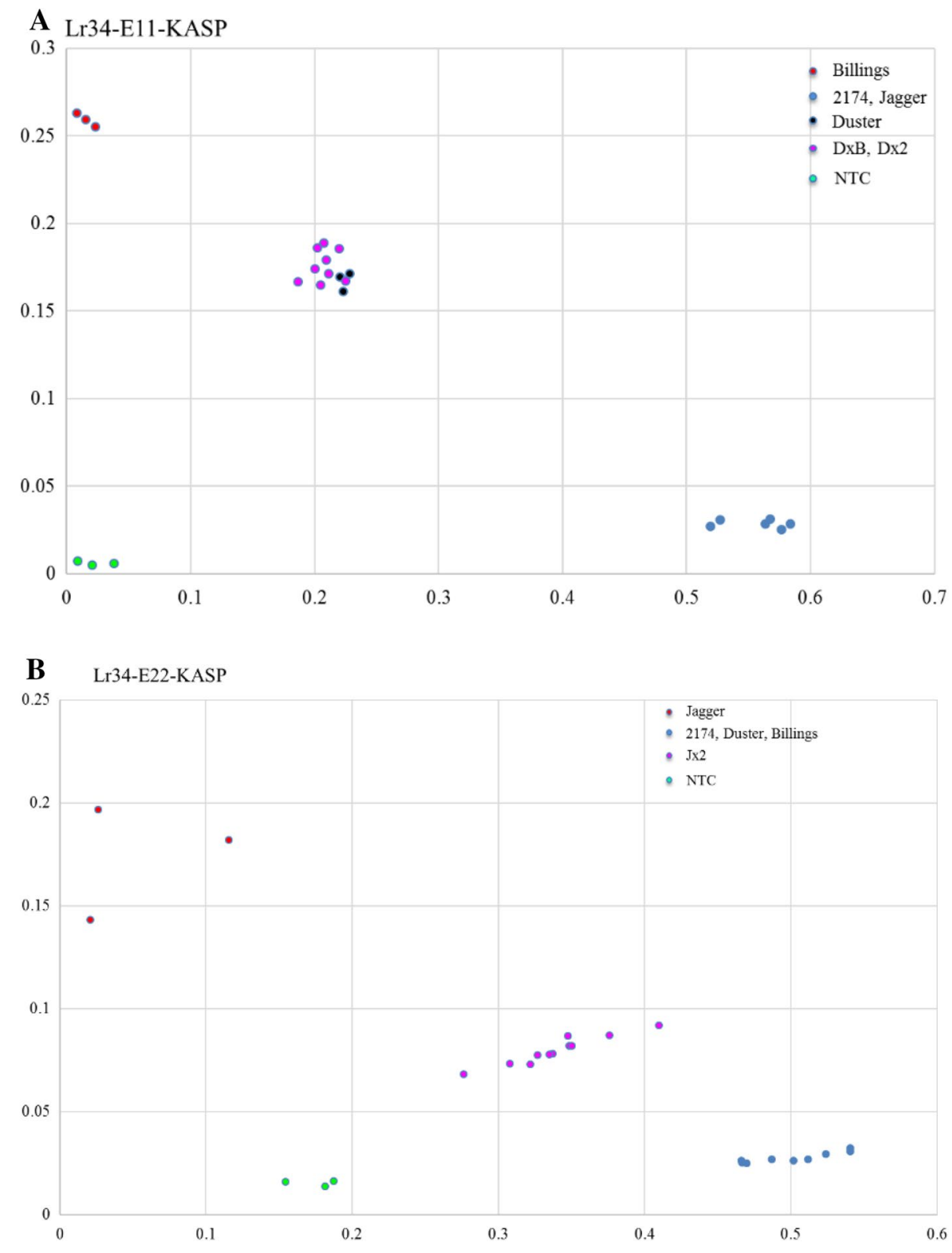

of each of $L r 34 a$ and $L r 34 b$ genes in Duster were mapped in the two independent populations, providing more convincing evidence for the existence of the $L r 34 a$ and $L r 34 b$ genes in a single genome in Duster. Lr34a in Duster has the In4r-E11r$E 12 r-E 22 r$ exon structure that is the same as CS, and $L r 34 b$ in Duster has In4s-E11s-E12s-E22r structure that is the same as Renan. Therefore, the haplotype of $L r 34 a b$ is novel.

A conventional approach to confirm the existence of duplicated DNA fragments or genes in a genome is to use $\mathrm{P}^{32}$-labled DNA probe or fluorescence in situ hybridization (FISH) to hybridize with a Southern blot of total gDNAs of the genome, and a conclusion is drawn based on the DNA band pattern and intensity of digested DNAs on the blot (Yan et al. 2004). A qRT-PCR method is also used to successfully determine copy number of genes in previous studies (Diaz et al 2012; Li et al. 2015). Since the wheat genome has been sequenced and the genes in a specific genome region are ordered, the qRT-PCR method can be used to determine if a single gene is duplicated or a genomic region containing the ordered genes is duplicated. The qRTPCR method is relatively easier and also provides convincing results compared with the conventional FISH method. We used the qRT-PCR method to determine copy number of neighboring genes adjacent to $\mathrm{Lr} 34$ in the reported BAC clone. The order of genes in the BAC clone containing Lr34 (Krattinger et al. 2009) is the same as annotated in the wheat genome sequence at IWGSC RefSeq v1.0. We concluded that the chromosomal fragment including Lr34 and at least two neighboring genes on its proximal side but excluding genes on its distal side occurred was duplicated in Duster. 
It is not yet known, however, how the chromosomal fragment duplication occurred. It is significant that the Lr34-B gene on homoeologous chromosome 7B was translocated into homoeologous chromosome 4A (Krattinger et al. 2011); therefore, three homoeologous $\operatorname{Lr} 34$ genes are located on homoeologous chromosomes $7 \mathrm{D}, 7 \mathrm{~A}$, and $4 \mathrm{~A}$. It was reported that there is clear chromosome distortion on the short arm of chromosome 7DS (Wang et al. 2009). It is possible that the chromosome structures in the region covering Lr34 of homoeologous chromosome 7 cause the translocation and the duplication. In the wheat literature, translocation events of $7 \mathrm{~B} / 4 \mathrm{~A}$ and $4 \mathrm{~A} / 5 \mathrm{~A}$ have been known for many years, but the mechanisms underlying the translocations are not yet known. Much more work is required to unravel the mystery.

It is also intriguing why the chromosomal fragment occurred in Duster, a unique winter wheat cultivar. It is also found that that Duster has the QYld. osu- $1 B S$ allele on chromosome arm 1BS that confers higher grain yield, which is a unique haplotype in the whole exome capture dataset, compared with 57 cultivars and breeding lines with various genetic backgrounds (Kan et al. 2020). It is not yet known where those unique sequences in Duster originated.

It was not surprising that the Duster Lr34ab haplotype was effective against leaf rust, since it has $L r 34 a$ that has identical sequence to Lr34e in 2174. Lr34e in 2174 was previously demonstrated to be effective when tested in Oklahoma (Cao et al. 2010). The linkage of Lr34ab genes in Duster with tip necrosis further supported the existence of the functional Lr34a in Duster. Even if a cultivar has a functional $L r 34$ gene, most of its transcripts were not completely spliced in a correct pattern, resulting in partial resistance of this gene (Fang et al. 2017). Therefore, the resistance of the Lr34 gene against leaf rust could be increased by eliminating or mutating regulators that cause mis-splicing events in wheat (Fang et al. 2017). Previous transformation studies on $L r 34 a$ showed that when the resistance gene $L r 34 a$ was transferred to a cultivar carrying the $L r 34 b$ gene, the transgenic wheat showed sufficient resistance to leaf rust compared to natural wheat containing an endogenous $\mathrm{Lr} 34 \mathrm{a}$ gene, demonstrating function of $L r 34 a$ in wheat (Risk et al. 2012; Rinaldo et al. 2017), barley (Boni et al. 2018), rice (Krattinger et al. 2016), Sorghum (Schnippenkoetter et al. 2017), and maize (Sucher et al. 2017). Transgenic wheat, however, has not yet been approved for commercial use. So far, the deployment of the Lr34a gene is still based on the conventional breeding approach, by which a resistant allele from a donor is introduced to a recipient cultivar carrying a susceptibility allele; therefore, only a single copy of the resistant $L r 34 a$ allele is yet achieved in a cultivar. The coexistence of $L r 34 a$ and $L r 34 b$ at the same locus in Duster provides an intriguing possibility that two natural resistance genes could be combined into a single genotype by conventional crossing methods, one from Lr34a in Duster and the other from 2174 or its descendent that carries the resistant gene $\mathrm{Lr} 34 \mathrm{e}$. The $\mathrm{CNV}$ resulting from the duplication of a gene is difficult to work with, because the native gene and the duplicated gene(s) are very similar in sequence (Dubcovsky and Dvorak 2007; Saintenac et al. 2011). It is even much more difficult to work with duplicated genes in bread wheat, as this species already has three homoeologous genes that have similar sequences.

Duster and its derivative have been elite genetic source in breeding programs of winter wheat in Oklahoma and surrounding states. Previous molecular markers for $\mathrm{Lr} 34$ were developed based on restriction enzyme digestion for detection of allelic variation in this gene. We attempted to develop KASP marker for each allelic variation intron 4, exon 11, exon 12 , and exon 22 , but the KASP system did not work for intron 4 or exon 12. Intron 4 is not involved in the function of Lr34, and exon 12 is always linked with exon 11 in their genotypes. The development of KASP markers for SNPs/ InDels at exon 11, as well as exon 22 of $L r 34$ would accelerate identification and deployment of the resistance alleles in wheat breeding.

Acknowledgements This work was supported by USDA-NIFA T-CAP Grant No. 2011-68002-30029, and the Oklahoma Center of Advanced Science and Technology (OCAST), the Oklahoma Wheat Research Foundation, and the Oklahoma Agricultural Experiment Station.

Author contribution statement T. Fang, L. Lei. G. Li, and C. Powers performed the experiments and analyzed data. B. Carver developed and phenotyped the population. L. Yan designed the experiments. T. Fang and L. Yan wrote the manuscript.

\section{Compliance with ethical standards}

Conflict of interest The authors declare that they have no conflict of interest.

Open Access This article is licensed under a Creative Commons Attribution 4.0 International License, which permits use, sharing, adaptation, distribution and reproduction in any medium or format, as long as you give appropriate credit to the original author(s) and the source, provide a link to the Creative Commons licence, and indicate if changes were made. The images or other third party material in this article are included in the article's Creative Commons licence, unless indicated otherwise in a credit line to the material. If material is not included in the article's Creative Commons licence and your intended use is not permitted by statutory regulation or exceeds the permitted use, you will need to obtain permission directly from the copyright holder. To view a copy of this licence, visit http://creativecommons.org/licenses/by/4.0/.

\section{References}

Boni R, Chauhan H, Hensel G, Roulin A, Sucher J, Kumlehn J, Brunner S, Krattinger SG, Keller B (2018) Pathogen-inducible Ta-Lr34res expression in heterologous barley confers disease 
resistance without negative pleiotropic effects. Plant Biotechnol J 16:245-253

Cao S, Carver BF, Zhu X, Fang T, Chen Y, Hunger RM, Yan L (2010) A single-nucleotide polymorphism that accounts for allelic variation in the $\mathrm{Lr} 34$ gene and leaf rust reaction in hard winter wheat. Theor Appl Genet 121:385-392

Chalupska D, Lee HY, Faris JD, Evrard A, Chalhoub B, Haselkorn R, Gornicki P (2008) Acc homoeoloci and the evolution of wheat genomes. Proc Natl Acad Sci USA 105:9691-9696

Dakouri A, McCallum BD, Walichnowski AZ, Cloutier S (2010) Fine-mapping of the leaf rust Lr34 locus in Triticum aestivum (L.) and characterization of large germplasm collections support the $\mathrm{ABC}$ transporter as essential for gene function. Theor Appl Genet 121:373-384

Diaz A, Zikhali M, Turner AS, Isaac P, Laurie DA (2012) Copy number variation affecting the photoperiod-B1 and vernalization-A1 genes is associated with altered flowering time in wheat (Triticum aestivum). PLoS ONE 7:e33234

Dubcovsky J, Dvorak J (2007) Genome plasticity a key factor in the success of polyploid wheat under domestication. Science 316:1862-1866

Dvořák J, DiTerlizzi P, Zhang H-B, Resta P (1993) The evolution of polyploidy wheats: identification of the A genome donor species. Genome 36:21-31

Edwards JT, Hunger RM, Smith EL, Horn GW, Chen MS, Yan L, Bai G, Bowden RL, Klatt AR, Rayas-Duarte P, Osburn RD, Giles KL, Kolmer JA, Jin Y, Porter DR, Seabourn BW, Bayles MB, Carver BF (2012) 'Duster' wheat: a durable, dual-purpose cultivar adapted to the southern Great Plains of the USA. J Plant Regist 6:37-48

Ellis JG, Lagudah ES, Spielmeyer W, Dodds PN (2014) The past, present and future of breeding rust resistant wheat. Front Plant Sci 5:641

Fang T, Campbell KG, Liu Z, Chen X, Wan A, Li S, Liu S, Cao S, Chen Y, Bowden RL, Carver BF, Yan L (2011) Stripe rust resistance in the wheat cultivar Jagger is due to $\mathrm{Yr} 17$ and a novel resistance gene. Crop Sci 51:2455-2465

Fang T, Carver BF, Hunger RM, Yan L (2017) Mis-Spliced Lr34 transcript events in winter wheat. PLoS ONE 12:e0171149

Huang S, Sirikhachornkit A, Su X, Faris J, Gill B, Haselkorn R, Gornicki P (2002) Genes encoding plastid acetyl-CoA carboxylase and 3-phosphoglycerate kinase of the Triticum/Aegilops complex and the evolutionary history of polyploid wheat. Proc Natl Acad Sci USA 99:8133-8138

Hunger RM, Edwards JT, Bowden RL, Yan L, Rayas-Duarte P, Bai G, Horn GW, Kolmer JA, Giles KL, Chen M-S, Jin Y, Osburn RD, Bayles MB, Seabourn BM, Klatt AR, Carver BF (2014) 'Billings' wheat combines early maturity, disease resistance, and desirable grain quality for the Southern Great Plains, USA. J Plant Regist 8:22-31

IWGSC (2018) Shifting the limits in wheat research and breeding using a fully annotated reference genome. Science 361:eaar7191

Kan C-C, Jia H, Powers C, Carver BF, Yan L (2020) Genetic characterization and deployment of a major gene for grain yield on chromosome arm 1BS in winter wheat. Mol Breed. https://doi. org/10.1007/s11032-020-1106-0

Kolmer JA (2017) Genetics of leaf rust resistance in the hard red winter wheat cultivars Santa Fe and Duster. Crop Sci 57:2500-2505

Kolmer JA, Su Z, Bernardo A, Chao S (2019) A backcross line of Thatcher wheat with adult plant resistance leaf rust resistance derived from Duster wheat has Lr46 and Lr77. Phytopathology 109:127-132

Krattinger SG, Lagudah ES, Spielmeyer W, Singh RP, Huerta-Espino J, McFadden H, Bossolini E, Selter LL, Keller B (2009) A putative $\mathrm{ABC}$ transporter confers durable resistance to multiple fungal pathogens in wheat. Science 323:1360-1363
Krattinger SG, Lagudah ES, Wicker T, Risk JM, Ashton AR, Selter LL, Matsumoto T, Keller B (2011) Lr34 multi-pathogen resistance $\mathrm{ABC}$ transporter: molecular analysis of homoeologous and orthologous genes in hexaploid wheat and other grass species. Plant J 65:392-4031

Krattinger SG, Jordan DR, Mace ES, Raghavan C, Luo M-C, Keller B, Lagudah ES (2013) Recent emergence of the wheat Lr34 multi-pathogen resistance: insights from haplotype analysis in wheat, rice, sorghum and Aegilops tauschii. Theor Appl Genet 126:663-672

Krattinger SG, Sucher J, Selter LL, Chauhan H, Zhou B, Tang M, Upadhyaya NM, Mieulet D, Guiderdoni E, Weidenbach D, Schaffrath U, Lagudah ES, Keller B (2016) The wheat durable, multipathogen resistance gene Lr34 confers partial blast resistance in rice. Plant Biotechnol J 14:1261-1268

Lagudah ES (2011) Molecular genetics of race non-specific rust resistance in wheat. Euphytica 179:81-91

Lagudah ES, McFadden H, Singh RP, Huerta-Espino J, Bariana HS, Spielmeyer W (2006) Molecular genetic characterization of the Lr34/Yr 18 slow rusting resistance gene region in wheat. Theor Appl Genet 114:21-30

Lagudah ES, Krattinger SG, Herrera-Foessel S, Singh RP, HuertaEspino J, Spielmeyer W, Brown-Guedira G, Selter LL, Keller B (2009) Gene-specific markers for the wheat gene Lr34/Yr18/ Pm38 which confers resistance to multiple fungal pathogens. Theor Appl Genet 118:889-898

Li G, Wang Y, Chen M, Edae E, Poland J, Akhunov E, Chao S, Bai G, Carver BF, Yan L (2015) Precisely mapping a major gene conferring resistance to Hessian fly in bread wheat using genotyping-by-sequencing. BMC Genom 16:108

Lillemo M, Joshi AK, Prasad R, Chand R, Singh RP (2013) QTL for spot blotch resistance in bread wheat line Saar co-locate to the biotropic disease resistance loci Lr34 and Lr46. Theor Appl Genet 126:711-719

McFadden ES, Sears ER (1946) The origin of Triticum spelta and its free-threshing hexaploid relatives. J Hered 37:81-89

Rinaldo A, Gilbert B, Boni R, Krattinger SG, Singh D, Park RF, Lagudah E, Ayliffe M (2017) The Lr34 adult plant rust resistance gene provides seedling resistance in durum wheat without senescence. Plant Biotechnol J 15:894-905

Risk JM, Selter LL, Krattinger SG, Viccars LA, Richardson TM, Buesing G, Herren G, Lagudah ES, Keller B (2012) Functional variability of the Lr34 durable resistance gene in transgenic wheat. Plant Biotechnol J 10:477-487

Risk JM, Selter LL, Chauhan H, Krattinger SG, Kumlehn J, Hensel G, Viccars LA, Richardson TM, Buesing G, Troller A, Lagudah ES, Keller B (2013) The wheat Lr34 gene provides resistance against multiple fungal pathogens in barley. Plant Biotechnol J 11:1467-7652

Saintenac C, Jiang D, Akhunov ED (2011) Targeted analysis of nucleotide and copy number variation by exon capture in allotetraploid wheat genome. Genome Biol 12:R88

Schnippenkoetter W, Lo C, Liu G, Dibley K, Chan WL, White J, Milne R, Zwart A, Kwong E, Keller B, Godwin I, Krattinger SG, Lagudah E (2017) The wheat Lr34 multipathogen resistance gene confers resistance to anthracnose and rust in sorghum. Plant Biotechnol J 15:1387-1396

Spielmeyer W, Mago R, Wellings C, Ayliffe M (2013) Lr67 and Lr34 rust resistance genes have much in common-they confer broad spectrum resistance to multiple pathogens in wheat. BMC Plant Biol 13:96

Sucher J, Boni R, Yang P, Rogowsky P, Büchner H, Kastner C, Kumlehn J, Krattinger SG, Keller B (2017) The durable wheat disease resistance gene Lr34 confers common rust and northern corn leaf blight resistance in maize. Plant Biotechnol J $15: 489-496$ 
Wang S, Carver BF, Yan L (2009) Genetic loci in the photoperiod pathway interactively modulate reproductive development of winter wheat. Theor Appl Genet 118:1139-1149

Wang GZ, Miyashita NT, Tsunewaki K (1997) Plasmon analyses of Triticum (wheat) and Aegilops: PCR single-strand conformational polymorphism (PRC-SSCP) analyses of organellar DNAs. Proc Natl Acad Sci USA 94:14570-14577

Yan L, Helguera M, Kato K, Fukuyama S, Sherman J, Dubcovsky J (2004) Allelic variation at the $V R N-1$ promoter region in polyploid wheat. Theor Appl Genet 109:1677-1686
Publisher's Note Springer Nature remains neutral with regard to jurisdictional claims in published maps and institutional affiliations. 